\title{
Le traitement des Roms dans l'Union Européenne
}

\section{Elspeth Guild}

Traducteur : Mathias Delori (depuis l'anglais)

\section{(2) OpenEdition \\ Journals}

\section{Édition électronique}

URL : http://journals.openedition.org/conflits/18145

DOI : $10.4000 /$ conflits. 18145

ISSN : $1777-5345$

Éditeur :

CCLS - Centre d'études sur les conflits lilberté et sécurité, L'Harmattan

Édition imprimée

Date de publication : 5 septembre 2011

Pagination : 192-194

ISBN : 978-2-296-56086-4

ISSN : 1157-996X

\section{Référence électronique}

Elspeth Guild, «Le traitement des Roms dans I'Union Européenne », Cultures \& Conflits [En ligne], 81-82 | Printemps/Été 2011, mis en ligne le 05 septembre 2012, consulté le 30 mars 2021. URL: http://journals.openedition.org/conflits/18145; DOI : https://doi.org/10.4000/conflits.18145 


\section{Le traitement des Roms dans I'Union Européenne *}

\section{Elspeth GUILD}

Elspeth Guild est Chercheur associée an Centre for European Policy Studies (CEPS), Professeur de Droit Européen des Migrations à l'Université de Nijmegen (Pays-Bas), et Professeur invitée à la London School of Economics (LSE).

T 'expulsion de Roms bulgares et roumains d'Italie en 2009 et de France en L2010 a provoqué de nombreuses discussions, contestations et préoccupations. En ce qui concerne la France, les expulsions de Roms de juillet et août 2010 (des expulsions seraient encore en cours selon de nombreuses sources) ont eu de multiples conséquences : elles ont généré de la discorde au sein des institutions de l’Union Européenne ; elles ont amené le commissaire européen chargé des droits des citoyens à s'opposer au président français; elles ont enfin provoqué de l'indignation au-delà de l'Union Européenne (UE). Curieusement, la question de l'effectivité et de l'efficacité de la politique d'expulsion n'a pas fait l'objet d'une attention particulière dans le débat politique. Pour combler cette lacune, il importe de dire un mot des objectifs de cette politique. Dans l'UE, l'expulsion d'individus est généralement entreprise afin de mettre un terme à la présence illégale d'individus sur le territoire d'un État, une intention souvent adossée à un objectif en termes de protection des populations ou de sécurité. Ceci implique deux idées :

- l'expulsion de la personne se trouvant dans une situation irrégulière dans un État quittera cet État, ce qui mettra un terme à sa présence non autorisée ;

- l'expulsion améliorera la gouvernance publique si la personne dont les activités (ou la simple présence) ont été déclarées illégales ne sera effectivement plus présente sur le territoire de l'État.

L'expulsion de ressortissants de Roumanie ou de Bulgarie par d'autres États membres de l'UE peut-elle être effective et efficace selon ces critères ?

* Traduction de l'anglais par Mathias Delori. 
De la réponse à cette question dépend la compréhension de ce qui se trame dans le débat sur les Roms.

Selon la Commission européenne, il y aurait dans l'UE entre 10 et 12 millions de Roms ressortissants d'un État membre. La plupart résident en Bulgarie, en Hongrie, en Roumanie et en Slovaquie. La population de ces pays représente 9,1 \% de la population totale de l'UE. En France, $2 \%$ de la population est composée de ressortissants d'autres pays de l'UE (selon Eurostat 94/2009). Dans l'ensemble, 5,4 \% des citoyens de l'UE ne vivant pas dans leur État membre sont des Roumains ; 44 \% d'entre eux vivent en Espagne et $37 \%$ en Italie. Parmi les étrangers vivant en France, les cinq principaux groupes sont les Portugais, les Algériens, les Marocains, les Turcs et les Italiens. Ces quelques données suffisent pour dissiper un fantasme. Contrairement à ce que l'on entend, le nombre total de Roms roumains en France n'est pas significatif. Dès lors, tout argument en termes d'effectivité ou d'efficacité qui mettrait en avant l'image d'une masse de Roms migrant vers la France est fallacieux.

Nous écrivions plus haut que l'effectivité et l'efficacité d'une politique publique se mesure à l'aune de ses objectifs. Dans le cas qui nous intéresse, ceci implique, premièrement, l'existence d'un mécanisme garantissant que les Roms roumains quittent bien le territoire français (mesures de reconduites à la frontière) et, deuxièmement, qu'ils ne sont pas capables d'y entrer de nouveau sans autorisation. En ce qui concerne la première condition, d'aucuns pourraient prétendre que cette politique fait montre d'une certaine effectivité et efficacité. Après tout, les médias nous inondent d'images de Roms embarqués dans des avions en France puis débarqués dans des aéroports roumains. Le problème devient plus aigu quand on considère le second aspect du critère d'effectivité et d'efficacité : la France a-t-elle les moyens de réguler l'éventuel retour des personnes expulsées?

Sur ce point, la réponse est un non catégorique. Les autorités françaises n'ont absolument aucun moyen d'empêcher les Roms roumains qu'ils viennent d'expulser de revenir en France dès qu'ils ont posé un pied sur le sol roumain. La raison est d'ailleurs peut-être plus pratique que juridique : les Roms roumains, qui détiennent des passeports roumains, sont des citoyens de l'Union. En tant que tels, ils ont le droit de se déplacer et de résider jusqu'à trois mois où ils le veulent dans l'UE, à moins que l'État soit en mesure d'opposer un argument extraordinaire motivé par des considérations politiques. En pratique, tous les États membres respectent le droit des citoyens de l'UE de se déplacer et de résider jusqu'à trois mois sur leur territoire. Même si on aimerait que cela fût le cas, les États ne suivent pas seulement cette ligne de conduite parce qu'ils tiennent à respecter leurs obligations vis-à-vis du droit communautaire ; ils adoptent cette posture car il n'existe aucun mécanisme empêchant les citoyens de l'UE de se déplacer et de résider où bon leur semble, en particulier au sein de la zone Schengen. Si un Rom roumain décide, 
juste après avoir été expulsé de France, de prendre un bus à Bucarest en direction de Paris, il ne devra montrer son passeport qu'à la frontière entre la Roumanie et la Hongrie. La suite de son trajet ne sera plus marquée par aucun contrôle d'identité. Il n'existe pas de garde-frontière à l'intérieur de la zone Schengen. Aucun fonctionnaire français ne sera donc amené à vérifier si le Rom roumain expulsé est revenu sur le territoire ou non.

D’une manière générale, les États de l'UE n'ont aucune idée du nombre de ressortissants d'un autre pays présents sur leur sol pendant moins de trois mois. Ces étrangers communautaires sont traités de la même manière que les Français qui se déplacent d'une région française à une autre. Après cette période, si un individu continue de résider sur le territoire, l'État peut exiger de lui qu'il obtienne un certificat d'inscription. Ceci étant, il suffit que cette personne rentre en Roumanie pour un week-end pour qu'une nouvelle période d'invisibilité débute.

Quand les États de l'UE expulsent un ressortissant d'un autre État membre, leur capacité à maintenir ces personnes hors de leur territoire est donc très limitée. Les outils administratifs permettant d'empêcher des ressortissants de l'UE d'entrer sur le territoire d'un autre État membre n'existent pas. Par ailleurs, le droit européen interdit aux États membres de mettre en place de tels outils. Ils ne pourraient pas, par exemple, remettre des gardes aux frontières à l'intérieur de l'espace Schengen. Les ressortissants de l'UE jouissent par ailleurs du droit d'entrer et de résider où bon leur semble dans n'importe quel État de l'UE. On dit parfois que la meilleure manière pour garantir des droits individuels consiste à placer les fonctionnaires dans l'incapacité pratique d'empiéter sur ces droits. C'est le cas pour les Roms roumains et la liberté de circulation.

Dès lors, quand on évalue l'effectivité et l'efficacité de la politique française d'expulsion des Roms roumains, l'objectif visant à maintenir certains individus en dehors du territoire français est mis à rude épreuve. Quelle que soit l'effectivité ou l'efficacité des expulsions, ces dernières ne comportent aucune garantie quant à la localisation des personnes le jour suivant. Dès lors, la compréhension de la politique d'expulsion des Roms passe peut-être par une réflexion supplémentaire sur son objectif. Si l'objectif affiché ne peut pas se traduire en termes d'effectivité et d'efficacité, on peut faire l'hypothèse qu'il en existe un autre. 